\title{
Endoscopic removal of accidentally ingested adult tooth brush
}

\author{
Sarfaraz Jasdanwala ${ }^{1 *}$, Avik Sarkar ${ }^{2}$, William Ferges $^{2}$ and Sita Chokhavatia ${ }^{2}$ \\ ${ }^{1}$ Department of Internal Medicine, Monmouth Medical Center, Long Branch, New Jersey, USA \\ ${ }^{2}$ Department of Gastroenterology, Robertwood Johnsons Rutgers Medical School, New Brunswick, New Jersey, USA
}

\begin{abstract}
Ingestion of foreign bodies and impaction of food boluses frequently require endoscopic intervention. The most common ingested foreign bodies include food, fish and chicken bones, medication packaging, dentures, and coins [1]. An adult sized toothbrush is infrequently ingested, and a literature review of prior cases reveals adults who ingest it often have psychiatric co-morbidities [2]. There are no reported cases of a toothbrush passing through the gastrointestinal tract spontaneously and removal is recommended to prevent complications. Endoscopic retrieval is recommended and if unsuccessful, a laparoscopic or surgical approach is recommended.

We present a 23 year old woman who reportedly accidently ingested an adult sized toothbrush. Initial attempt at endoscopic retrieval was unsuccessful due to inability to traverse the toothbrush past the gastro-esophageal junction. Subsequently with the use of a long over- tube, successful endoscopic retrieval was achieved. The patient tolerated both procedures well and was discharged home subsequently.

This case illustrates the importance of using a longer $(45 \mathrm{~cm})$ over-tube that extends beyond the gastro-esophageal junction, in endoscopic retrieval of ingested long foreign bodies such as a toothbrush. A review of the literature about the timing and techniques of endoscopic removal of ingested long foreign bodies as well as complications will be discussed.
\end{abstract}

\section{Case Presentation}

A 23 year old woman with no past medical history presented to the emergency department after accidentally swallowing an adult sized toothbrush. After eating excess amount of food at a party on the evening of admission, she was uncomfortable with the large volume of food ingested and reportedly used the back of an adult sized 7.5inch toothbrush to induce emesis. During this process, she reported losing control of the object and accidentally swallowed the adult sized toothbrush. The patient denied subjective complaints including chest and abdominal pain, discomfort, nausea or vomiting. She did not feel the toothbrush getting stuck at any point and reported it passed without difficulty. There was no reported past history of eating disorders. She denied any suicidal ideation.

Past medical history, family history and social history were reviewed and found to be unremarkable.

Physical exam was unremarkable. The abdomen was soft and nontender. There was no parotid gland enlargement or erosions of the lingual surface of the teeth, loss of enamel, periodontal disease, and extensive dental caries.

Labs including complete blood count, basic metabolic panel, prothrombin time (PT) and partial thromboplast intime (PTT) were within normal limits.

$\mathrm{X}$-ray Abdomen showed the toothbrush head bristles within the gastric fundus (Figure 1). X-ray of the neck revealed normal soft tissue shadow and patent airways.

Decision was made to retrieve the toothbrush endoscopically. Initial endoscopic retrieval was attempted in the operating room at midnight using polypectomy snare and rat tooth forceps but this was unsuccessful due to inability to introduce the toothbrush into the esophagus. The toothbrush was visualized in the fundus in a pool of residual liquid and solid food material. The bristled end was facing the pylorus and therefore the snare was used to rotate the toothbrush so that the bristled end was now proximal. The snare was firmly on the bristles but the toothbrush did not traverse through the gastroesophageal (GE) junction, despite different maneuvers including

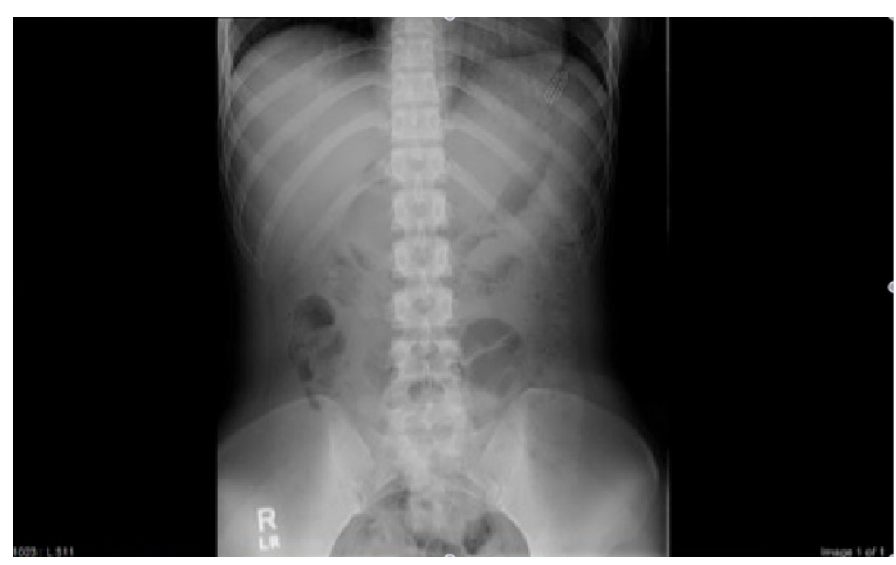

Figure 1. X-ray abdomen showing toothbrush bristles.

Correspondence to: Sarfaraz Jasdanwala, Department of Internal Medicine, Monmouth Medical Center, Long Branch, New Jersey, USA, Tel: 732-923-5000, ext 1450; E-mail: s.a.jasdanwala@gmail.com

Received: April 06, 2015; Accepted: May 04, 2015; Published: May 07, 2015 


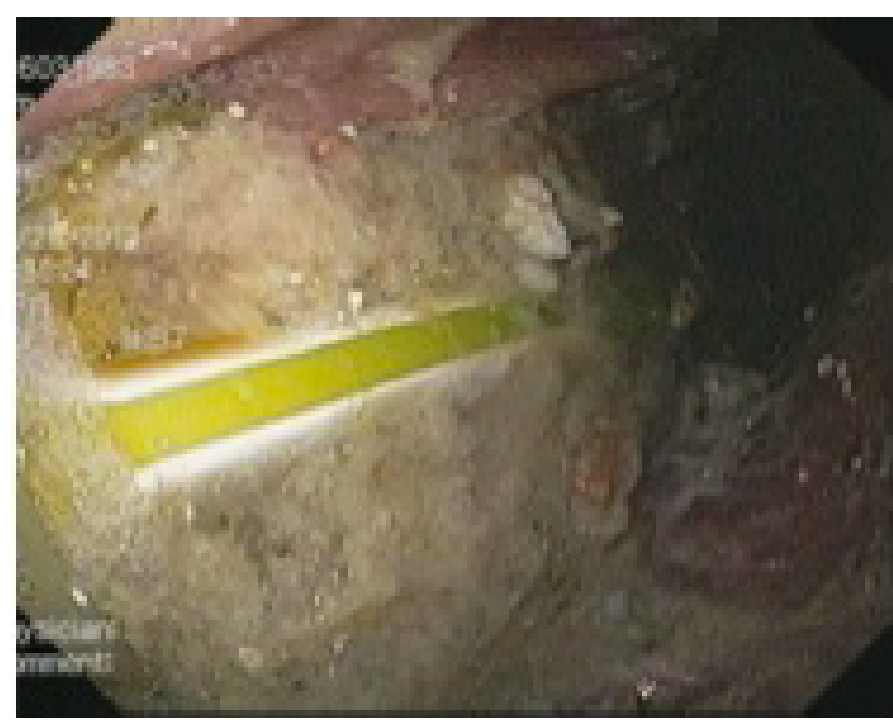

Figure 2. Toothbrush in stomach.

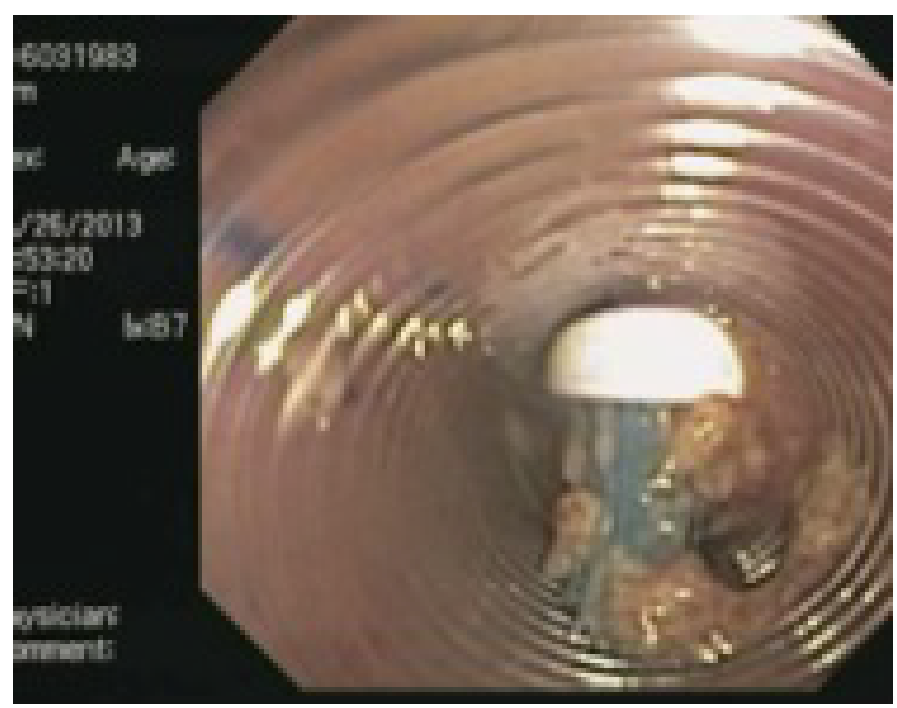

Figure 3. Toothbrush in the esophagus within the over tube.

Glucagon. The procedure was abandoned after 2 hours. A subsequent attempt was made the following morning using a long flexible overtube. A two channel (T2) endoscope was utilized and with a snare the endoscopist was able to grab the bristled end of the toothbrush and drag it into the over-tube. Once in the over-tube, the bristles of the toothbrush were grabbed with a rat tooth forceps and the toothbrush was successfully removed from the patient. The second procedure also took two hours, mostly due to the presence of a significant amount of food making visualization and maneuvering difficult (Figures 2 and 3).

The patient did well post procedure and was discharged to home later on the same day after being monitored under observation for 10 hours.

\section{Discussion}

Ingestion of foreign bodies and impaction of food bolus are not rare. An adult size toothbrush, quite surprisingly, is not an infrequently ingested foreign body [3]. A literature search found 40 published cases between 1988 to 2000 discussing endoscopic retrieval of an ingested adult sized toothbrush [4]. American Society for Gastrointestinal Endoscopy (ASGE) has devised guidelines for management of ingested foreign bodies and food impaction. These guidelines along with comprehensive review of literature about the various techniques that have been successfully employed in retrieving an ingested toothbrush will be discussed.

Toothbrush ingestions can be accidental or intentional. It is usually intentional in adults and is commonly accompanied by psychiatric co-morbidities such as bulimia or with impaired swallowing due to severe dementia or alcohol intoxication [2]. The clinical diagnosis is easily made on the basis of history and plain films of the abdomen. The incidence of perforation is not common. Ulcerations or erosions are more common [3]. However, duodenal perforation from an ingested toothbrush requiring surgical intervention has been reported [5]. As per the American Society for Gastrointestinal Endoscopy recommendations, removal of any foreign body greater than $6 \mathrm{~cm}$, including toothbrushes, should be performed as soon as possible, with a vast majority of them being removed within 48 hours [6]. This recommendation is based on studies showing that $>80 \%$ of foreign bodies $>6 \mathrm{~cm}$ in length did not pass through the pylorus at the time of performing endoscopy [2].

The technique for removal requires sedation, use of the proper equipment, and knowledge of the modalities available. It is recommended that at least 3 nurses or assistants be available for help during the procedure [7]. The ASGE guidelines recommend the use of glucagon for impacted esophageal food bolus despite lack of clear evidence, as it is relatively safe. Mechanism of action of glucagon in this setting is to relax the lower esophageal sphincter making it easier for the food bolus to pass through the esophagus [8]. This recommendation is not specifically made for long foreign objects like toothbrush. Review of previously published cases shows that glucagon was not used prior to endoscopy. In our case, we used $1 \mathrm{mg}$ glucagon intravenously during the first procedure to relax the lower esophageal sphincter so as to facilitate easy retrieval from the gastro-esophageal junction. Despite this, the toothbrush was not able to be drawn into the esophagus.

Use of a long flexible over-tube $>45 \mathrm{~cm}$ which can cover the distal gastro esophageal (GE) junction is strongly recommended in endoscopic retrieval of ingested long foreign bodies [6,9]. The most technically challenging part of the procedure is maneuvering the tip of the toothbrush into the GE junction without letting the bristles slip off. Use of a long overt-tube greatly facilitates this [7]. We used a long over tube during the second attempt. Our anesthesiologists recommend that the patient be intubated prior to the passage of over-tube as it may be difficult to intubate the patient once the over-tube is in place. Also this may help prevent compression on trachea.

The recommendations vary for the use of the retrieval tool. Several authors recommend the use of dormia basket instead of polypectomy snare as the bristles slip off from the snare $[9,10]$. However several authors have used polypectomy snares alone or in conjunction with rat tooth forceps for successful removal. The ASGE guidelines recommend the use of either snare or basket [5,6]. In our experience the use of snare along with rat tooth forceps was convenient. We preferred the use of snare due to the easier availability and greater endoscopist experience with snare as compared to basket for foreign body retrieval. Practicing grasping the toothbrush outside with the same tool before the procedure is helpful.

The toothbrush is commonly found to be perpendicular to the esophageal axis within the stomach. It could be parallel to the long axis 
too with the bristled end facing either proximally or distally. Regardless of the initial orientation, it is recommended that the toothbrush be maneuvered so that the long axis of the toothbrush be in parallel to the long axis of the esophagus with the bristled end lying proximally. This is the easiest position for retrieval. Grasping the toothbrush with the handle is difficult as the handle is slippery. Applying external pressure on the stomach or changing the position of the patient can help with grasping of the toothbrush [7]. In our case, the toothbrush was fortunately lying parallel to the long axis of the esophagus with the bristled end facing proximally. However there was presence of a retained food in the stomach making the grasping of the bristles difficult.

Once successful grasping has been accomplished, the upper endoscope with the toothbrush should be pulled into the over- tube. Once the toothbrush is in the over- tube it is recommended that the over-tube along with the toothbrush and the endoscope should be withdrawn together in a single movement [6]. In our case, the endoscopist used rat tooth forceps once the toothbrush was within the over-tube for better grasp. The tooth brush was pulled out using the rat tooth forceps. The over- tube was subsequently withdrawn.

Acid suppression therapy can be used if erosions have developed as a complication. In our case, second look revealed no esophageal or gastric erosions.

Not all types of toothbrushes can be retrieved endoscopically. The type of toothbrushes that are unlikely to be successfully removed include ones that have angulated handles or ones with bristles that come out easily making it impossible to grasp the toothbrush.

Recommendations for surgical removal can be made if the endoscopic retrieval is unsuccessful or if there is presence of complications like perforation. Laparoscopic gastrostomy is the preferred surgical approach [11].

\section{Conclusion}

An ingested adult sized toothbrush cannot pass through the gastrointestinal tract without intervention. Therefore, it is mandatory that it should be retrieved endoscopically. This is technically very demanding. In order to reduce the incidence of complications including intestinal perforation and intestinal obstruction, it should be done on an urgent basis. However, it is not always possible to successfully remove a toothbrush endoscopically. If this fails, or if complications develop, then surgical approach is recommended.

\section{References}

1. Wu WT, Chiu CT, Kuo CJ, Lin CJ, Chu YY, et al. (2011) Endoscopic management of suspected esophageal foreign body in adults. Dis Esophagus 24: 131-137. [Crossref]

2. Palta R, Sahota A, Bemarki A, Salama P, Simpson N, et al. (2009) Foreign body ingestion: characteristics and outcomes in a lower socioeconomic population with predominantly accidental ingestion. Gastrointest Endosc 69: 426-33. [Crossref]

3. Kirk AD, Bowers BA, Moylan JA, Meyers WC (1988) Toothbrush swallowing. Arch Surg 123: 382-384. [Crossref]

4. Faust J, Schreiner O (2001) A swallowed toothbrush. Lancet 357: 1012. [Crossref]

5. Chao HH1, Chao TC (2008) Perforation of the duodenum by an ingested toothbrush. World J Gastroenterol 14: 4410-4412. [Crossref]

6. ASGE Standards of Practice Committee, Ikenberry SO, Jue TL, Anderson MA, Appalaneni V, et al. (2011) Management of ingested foreign bodies and food impactions. Gastrointest Endosc 73: 1085-1091. [Crossref]

7. Chinitz MA, Bertrand G (1990) Endoscopic removal of toothbrushes. Gastrointest Endosc 36: 527-530. [Crossref]

8. Sodeman TC, Harewood GC, Baron TH (2004) Assessment of the predictors of response to glucagon in the setting of acute esophageal food bolus impaction. Dysphagia 19: 18-21. [Crossref]

9. Vantsian EN, Krendal AP (1984) Two rare cases of endoscopic extraction of foreign bodies from the stomach and duodenum. Endoscopy 16: 242-245. [Crossref]

10. Bastos I, Gomes D, Cotrim I, Gouveia H, Donato A, et al. (1996) An unusual endoscopic procedure to remove a toothbrush from the stomach. Endoscopy 28: 525. [Crossref]

11. Harvey M, Cave G, Prince G (2012) Endoscopic removal of an inadvertently swallowed toothbrush in the emergency department. Case Rep Emerg Med 2012 568163. [Crossref]

Copyright: (C2015 Jasdanwala S. This is an open-access article distributed under the terms of the Creative Commons Attribution License, which permits unrestricted use, distribution, and reproduction in any medium, provided the original author and source are credited. 